$$
\begin{gathered}
\text { 간호 시뮬레이션 교육이 간호대학생의 문제해결과정과 } \\
\text { 자기효능감에 미치는 효과 }
\end{gathered}
$$

오혜경, 한영인

춘해보건대학교 간호학과

\title{
Effects of Nursing Simulation-Based Education on Problem Solving Process and Self-efficacy of Nursing College Students
}

\author{
Hye-Kyung Oh, Young-In Han \\ Department of Nursing, Choonhae Health Science College
}

\begin{abstract}
$<$ Abstract $>$
The purpose of this study was to approve the effects of nursing simulation-based education on problem solving process and self-efficacy for nursing students. The nursing students of 244 participated the nursing simulation-based education of 60 hours. The questionnaire survey on problem solving process and self-efficacy were conducted 2 time(before education, after education) to the nursing students. The data were analyzed by the SPSS 12.0 program. The results were as follows; There was a statistically significant increase in problem solving $\operatorname{process}(t=2.637, p=.012)$ but no statistically significants self-efficacy $(\mathrm{t}=0.135, \mathrm{p}=.743)$ effects of nursing simulation-based training in nursing college students. There was a significant positive correlation between problem solving process and self-efficacy $(r=0.737, p=.017)$. In conclusion, the study found that nursing simulation-based education for nursing students may increase problem solving process but no effective self-efficacy.
\end{abstract}

Key Words : Nursing Simulation-Based Training, Problem Solving Process, Self-Efficacy 


\section{I . 서론}

\section{1. 연구의 필요성}

최근의 의료 환경은 정보의 다양화와 대상자들 의 지식수준의 증가로 간호사에게 양질의 간호수 행과 숙련된 기술을 요구하고 있으며, 학생들은 임 상에서 다양한 술기를 경험하고 싶지만 의료소비 자들의 인식변화와 대상자요구 확대로 직접간호를 수행하기 어려운 실정에 있다[1]. 이러한 상황에서 간호사는 안전하고 전문적인 간호를 제공하기 위 해 좋은 문제 해결자가 되어야 하므로[2], 시대에 부응하는 간호사를 양성하기 위해서 변화에 민감 하게 대처할 수 있도록 조직적이고 탐구를 통한 학습방법이 필요하다[3]. 최근 시뮬레이터를 활용 한 교육은 실제적이고 상호작용적인 교수 학습방 법으로 전통적인 강의 방식의 교육보다 간호학생 의 지식과 기술을 향상시키고[4], 대상자에게 안정 과 안위를 고려하여 위해를 가하지 않고도 학습자 가 이론과 실제를 통합할 수 있는 학습환경을 제 공한다[5]. 시뮬레이션의 가장 큰 장점은 환자의 안전을 위협하지 않고도 피드백, 반복학습 그리고 재교육으로 연결될 수 있고[6], 학습자의 불안감 해소, 동료의 지지와 강의내용과 일관된 임상경험, 지식, 기술, 태도 영역에 대한 학습, 학생이 실습 시 접근하지 못했던 사례도 가능하다고 하였다[7]. 시뮬레이션 교육은 제한된 임상환경에서 구조화된 시나리오에 따라 구체화된 학습기회를 제공하는 교육으로[8], 컴퓨터 프로그램에 의해 생리반응을 일으키는 실제 임상환경에서 상황에 맞는 적절한 간호중재 능력과 비판적 사고, 문제해결능력을 증 진시키는 교육이다[9].

문제해결력은 바람직한 목표 상태에 도달하기 위한 것으로 어떤 행동의 결과라기보다는 과정적 인 의미를 가지며, 문제해결은 단계적인 과정을 거
치면서 획득되는 것을 말한다[10]. 합리적인 문제 해결을 위해서는 문제의 본질을 이해하고, 관련 정 보를 수집하며, 최선의 대안을 선택한 후 의사결정 시 효율적으로 판단하고 수행하여 검토하는 문제 해결과정이 요구된다[11]. 문제해결과정이란 목표 지향적인 일련의 조직활동으로 다양한 인지능력을 요구하는 사고활동의 표현이며, 지식과 정보를 탐 색, 선택, 조직하여 문제를 인식하고 해결대안을 고안하며 이를 적용한 후 그 결과를 평가하는 과 정을 말한다[12]. 시뮬레이션 실습교육은 간호학생 들의 비판적 사고성향, 문제해결력 증진에 효과적 이라고 하였으며[13], 팀기반 시률레이션 학습 프 로그램이 간호학생의 대인관계이해도, 문제해결능 력 및 학업성취도 향상을 촉진시키는데 매우 효과 적이라고 하였다[14]. 간호학생을 시뮬레이션기반 심폐응급간호교육을 적용한 결과, 실험군의 학업성 취도, 자기효능감, 학습태도, 수업만족도 간에 상관 관계가 있음을 보고하였다[15]. 간호대학생을 임상 실습교육을 적용한 결과 조직적이고 체계적인 임 상실습교육을 적용함으로써 간호기술을 효율적으 로 습득하여 졸업 후 전문직 간호사로서의 만족도 를 향상시키게 될 것이고[16], 수술실 간호사는 병 동간호사보다 자기효능감이 높으며, 자기효능감이 높을수록 의료기술이 높다고 하였다[17]. 자기효능 감은 Bandura의 자기효능이론에 의하면 '자기효능 감'(self-efficacy)은 성공적 수행에 필요한 행동적, 인지적, 정서적 자원을 선택적으로 동원하여 “어떤 종류의 수행을 실행하고 조직화하는 자신의 역능 에 대한 판단"으로 정의하였으며[18], 자기효능감 이 높은 사람의 특징으로는 어려운 일을 맡았을 때에 기꺼이 자신을 개입시키고 헌신하려는 경향 이 있다[19]고 하였다. 그러므로 본 연구는 간호 시뮬레이션 교육을 적용하여 간호학생의 문제해결 과정과 자기효능감에 미치는 효과를 규명하고자 하였다. 
2. 연구목적

본 연구는 간호대학생의 문제해결과정과 자기효 능감에 미치는 영향은 교육방법에 따라 달라질 수 있으므로 간호 시뮬레이션 교육이 간호대학생의 문제해결과정과 자기효능감에 미치는 영향를 규명 하고자 하였으며, 본 연구의 구체적인 목적은 다음 과 같다.

1) 간호 시뮬레이션 교육이 간호대학생의 문제 해결과에 미치는 효과를 파악한다.

2) 간호 시뮬레이션 교육이 간호대학생의 자기 효능감에 미치는 효과를 파악한다.

3) 간호대학생의 문제해결과정과 자기효능감 간 의 관계를 파악한다.

\section{П․ 연구방법}

\section{1. 연구 설계}

본 연구는 단일군 전후 실험설계로서 간호시뮬 레이션 교육전에 일반적 특성, 문제해결과정, 자기 효능감을 측정하였고, 실험처치 후에는 문제해결과 정, 자기효능감을 반복 측정하였다.

\section{2. 연구대상 및 자료수집}

본 연구대상자는 $\mathrm{U}$ 시에 있는 $\mathrm{C}$ 대학 간호과 간 호시뮬레이션 교육과정 15 팀 중에 연구자가 운영 하는 12 팀(288명)을 대상자를 편의표집하여 연구의 목적 및 방법, 연구참여에 대한 익명성 보장, 자발 적인 연구 참여 동의와 거부, 중도 포기가능, 발생 가능한 이익과 불이익 등을 포함한 내용를 설명한 후 본 연구에 자발적으로 참여하기로 동의한 연구 대상자를 선정하였으며 선정된 연구대상자는 244 명이었다. 자료수집기간은 2010년 3월 2일부터 2010년 6월 11일까지 총 105일간 자료수집하였다.
2010년 3월 2일에서 3월 9일까지 실험처치인 간호 시뮬레이션 교육 전에 사전조사 하였고, 간호시뮬 레이션 교육은 매주 4 시간씩 15주간 60시간 진행 하였으며, 사후평가는 간호시뮬레이션 교육 종합평 가 종료 직후 2010년 6월 2일에서 6월 11일까지 진행하였으며, 설문지는 대상자가 직접 설문지를 읽고 응답하였으며, 설문지 작성에 소요된 시간은 10 20분간 소요되었다.

\section{3. 연구도구}

본 연구에서 사용한 연구도구는 다음과 같다.

1) 간호시뮬레이션 교육

재현된 임상환경에서 구조화된 시나리오에 따라 구체화된 학습기회를 제공하는 교육으로시뮬레이 션 과정은 브리핑(briefing), 시뮬레이션, 디브리핑 (debriefing)으로 이루어진다[8]. 본 연구에서의 간 호시뮬레이션 교육은 연구자가 개발한 활력징후, 신체사정, 치료적 의사소통, 흡인법, 산소요법, 체 위변경, 투약, 통증, 수면장애, 상처간호 개념이 포 함된 현장사례중심모듈을 활용한 자기주도학습, 토 론식 수업과 시뮬레이터(SimMan 3G)를 이용한 임 상상황실습이 포함된 교육을 의미한다. 간호시뮬레 이션 교육은 팀 단위로 진행하였으며, 1 팀에 전임 교수 1명, 조교 1명, 학생 24명으로 12팀을 대상으 로 연구자 2 인과 조교 1 인이 본 연구에 참여하였 다. 간호시뮬레이션 교육의 일관성을 도모하기 위 해 일별, 주별 미팅을 실시하였으며, 교육 내용은 다음과 같이 진행하였다.

- 학습목적 : 간호시뮬레이션 교육은 임상에서 발생하는 대상자의 다양한 상황을 Simulator를 통 해 구현할 수 있으며, 안전하면서도 실제와 같은 상황에서 간호과정을 적용한 간호를 수행하게 함 으로써 학습의 효과를 증대시키는 교육방법이다. 재현된 임상환경에서 건강문제 해결을 위해 의사 
결정을 촉진할 수 있으며, 즉각적인 피드백과 간호 행위들에 대한 관찰, 체험학습, 성찰학습을 할 수 있고, 필요시 반복학습을 통해 정확하고 완성도 높 은 간호수기를 적용할 수 있다.

- 학습목표 : 의료기술의 발달과 간호대상자의 인식의 변화로 인해서 임상실습을 할 수 있는 대 상자의 감소와 위험하고 어려운 술기에 대한 효율 적인 훈련 요구의 증가, 환자 안전에 대한 요구도 증가, 의료사고의 예방, 복잡한 의료상황에서 건강 문제 해결을 위해 간호현장에서 능숙하게 적용할 수 있는 간호실무능력을 함양하도록 한다.

- 실습 교육내용 : 주별 간호시뮬레이션 교육 내 용은 다음과 같다.
2) 기본수기 평가도구

본 연구에서 학교성적은 간호시뮬레이션 교육 중에 기본수기 사전학습 전단계 시험 점수를 말하 며, 기본수기는 본 대학의 간호시뮬레이션 교과목 의 교재[20]를 참고하여 간호시뮬레이션 교수 2 인 과 연구자가 작성한 평가도구로 활력징후, 신체사 정, 치료적 의사소통, 흡인법, 산소요법, 체위변경, 투약, 통증, 수면장애, 상처간호에 대한 10 문항으로 구성되어 있으며 점수의 범위는 0 점에서 10 점으로 매우 우수 9점 이상, 우수 8점 6점, 낮음 5점-4점, 매우 낮음 3점 이하로 점수가 높을수록 학교성적 이 높은 것을 의미한다.

\begin{tabular}{|c|c|c|}
\hline 주별 & 주요내용 & 세부내용 \\
\hline 1 & 오리엔테이션 & - 모듈 소개, 사전학습 제시 \\
\hline 2 & $\begin{array}{l}\text { 기본수기 } \\
\text { 사전시험 }\end{array}$ & $\begin{array}{l}\text { - 기본수기 시험 시행(이론과 실기 시험 : 10점) } \\
\text { - 사전학습 }\end{array}$ \\
\hline 3 & 심맨 사용법 & - 심맨 사용법 숙지 : 호흡기계 이론(해부와 질환) 강의 \\
\hline 4 & 모듈 1 개발 & $\begin{array}{l}\text { - 모듈 } 1 \text { 개발 : 호흡기계 관련 환자 사례 } \\
\Rightarrow> \\
\text { - 사정 및 중재, 조별 토론하기 } \\
\text { - 모듈 } 1 \text { 개념(활력징후, 신체사정, 치료적 의사소통, 흡인법, 산소요법) }\end{array}$ \\
\hline 5 & 러닝 1 & - 모듈 1 시뮬레이션 실제 운용 \\
\hline 6 & 브리핑 1 & - 시뮬레이션 실제 운용 후 브리핑 \\
\hline 7 & 디브리핑 1 & $\begin{array}{l}\text { 종합 시뮬레이션 실습 } 1 \\
\text { 디브리핑 : 동영상 보여주면서 조별로 토론 } \\
\text { 디브리핑의 3단계 구술 } \rightarrow \text { 분석 } \rightarrow \text { 적용 }\end{array}$ \\
\hline 8 & & 중간고사 \\
\hline 9 & 모듈 2 개발 & $\begin{array}{l}\text { 모듈 } 2 \text { 개발 : 심장질환 문제를 가진 개방창상 환자 사례 } \\
\quad \Rightarrow \text { 사정 및 중재, 조별 토론하기 } \\
\text { - 모듈 } 2 \text { 개념(체위변경, 투약, 통증, 수면장애, 상처간호) }\end{array}$ \\
\hline 10 & $\begin{array}{l}\text { 러닝 } 2 \\
\text { 브리핑 }\end{array}$ & $\begin{array}{l}\text { 종합 시뮬레이션 실습 } 2 \\
\text { 모듈 2: 심장릴환 문제를 가진 개방창상 환자 시뮬레이션 실행 } \\
\text { 브리핑 }\end{array}$ \\
\hline 11 & $\begin{array}{l}\text { 모듈 } 3 \text { 개발 } \\
\text { 러닝 } 3\end{array}$ & $\begin{array}{l}\text { 러닝 } 3: \text { 모듈 1, 2를 합친 사례 } \\
\text { 브리핑 }\end{array}$ \\
\hline 12 & $\begin{array}{l}\text { 종합실습평가 } \\
\text { 사후시험 }\end{array}$ & $\begin{array}{l}\text { - 모듈 } 3 \text { 시뮬레이션 실행 } \\
\text { - 사후시험 : 이론과 실기 Test : 10점 }\end{array}$ \\
\hline 13,14 & 브리핑 & $\begin{array}{l}\text { 종합 시뮬레이션 실습 } 3 \text { (소그룹) } \\
\text { 디브리핑 : 동영상 보여주면서 조별로 토론 } \\
\text { 디브리핑의 3단계 구술 } \rightarrow \text { 분석 } \rightarrow \text { 적용 }\end{array}$ \\
\hline 15 & & 기말고사 \\
\hline
\end{tabular}


3) 문제해결과정

문제해결과정은 일상생활이나 혹은 특정 상황에 서 어떤 문제가 주어지거나 또는 직면하게 되는 문제를 해결하고자 할 때 문제의 본질이 무엇인지 명료화하고, 해결방안을 모색하고 그에 근거하여 의사결정을 한 다음 해결책을 수행하고 수행한 결 과에 대한 평가하고 반영하는 문제해결과정을 이 행하는 것을 의미한다[11]. 본 연구에서는 이우숙 등[11]이 개발한 문제해결과정 도구로 총 30 문항으 로 5점 Likert척도로 개발된 도구이다. 문제해결과 정은 5 단계(문제의 명료화 6 문항, 해결방안모색 6 문항, 의사결정 6 문항, 해결책 수행 6 문항, 평가 및 반영 6문항)로 분류되어 있으며 점수의 범위는 30 점에서 150점으로 점수가 높을수록 문제해결과정 이 높음을 의미한다. 개발 당시 Cronbach's $a=$ .93이었으며, 본 연구에서의 신뢰도는 Cronbach's $a=.91$ 이었다.

4) 자기효능감 측정도구

자기효능감은 어떤 과제를 특정 수준까지 해낼 수 있다는 개인의 판단이며 결과를 얻고자하는 행 동을 성공적으로 수행해낼 수 있다는 개인의 신념 으로 상황적·구체적 자신감의 강도를 의미한다 [19]. 본 연구에서는 Sherer 등[21]이 개발한 자기 효능감 측정도구를 Hong[22]이 번안한 도구로 총 23문항으로 5점 Likert척도로 개발된 도구이다. 자 기효능감은 일반적 자기효능감 요인 17 문항, 사회 적 자기효능감 요인 6 문항으로 분류되어 있으며 점수의 범위는 23점에서 115점으로 점수가 높을수 록 자기효능감이 높음을 의미한다. 개발 당시 Cronbach's $a=86$ 이었으며, 본 연구에서의 신뢰 도는 Cronbach's $a=.82$ 이었다.

\section{4. 자료분석방법}

수집된 자료는 SPSS WIN 12.0 프로그램을 이용
하여 통계분석 하였다.

1) 대상자의 일반적 특성은 간호시뮬레이션 실 습 전후의 빈도, 백분율 및 평균과 표준편차를 이 용하여 분석하였고, 측정도구의 신뢰도는 Cornbach's a로 분석하였다.

2) 간호 시뮬레이션 실습이 간호대학생의 문제 해결과정과 자기효능감에 미치는 영향을 알아보기 위해 가설검증은 paired t-test를 실시하였다.

3) 간호 시뮬레이션 실습 실시한 후에 간호대학 생들의 문제해결과정과 자기효능감 간의 상관관계 는 Pearson Correlation으로 분석하였다.

\section{5. 연구의 제한점}

본 연구는 단일군 전후 실험설계이며, 일 개 대 학의 간호과 2학년 중 간호시뮬레이션 실습 교육 과정에 참여한 일부팀원을 본 연구 대상으로 하였 으므로 확대 해석하기에는 제한점이 있다.

\section{III. 연구결과}

\section{1. 대상자의 일반적 특성}

연구 대상자의 일반적 특성은 성별로는 여학생 228 명 $(93.4 \%)$, 남학생 16 명 $(6.6 \%)$ 이었으며, 연령은 평균 22.24 \pm 3.09 세이었으며, 19세-21세 170명 $(69.7 \%)$ 로 가장 많았고, 25 세 이상 38 명(15.6\%), 22-24세 36명(14.8\%) 순으로 많았다. 종교는 기독교 125 명(51.2\%), 불교 97명(39.8\%), 천주교 20명 $(8.2 \%)$, 종교가 없는 경우 2 명 $(0.8 \%)$ 순이었다. 결 혼유무는 미혼이 172 명 $(70.5 \%)$, 기혼은 72 명 $(29.5 \%)$ 이었다. 자신의 생활에 대한 만족 정도는 평균 $3.85 \pm 1.54$ 이었으며 자신의 생활에 만족한다가 156 명 $(63.9 \%)$ 로 가장 높았다. 학교성적의 사전평균 은 $4.48 \pm$.79점이었으며, 학교성적이 낮다가 122 명 (50\%), 우수하다가 110 명(45.1\%)순이었고, 사후평균 


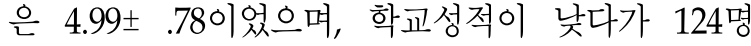
(51.3\%), 우수하다가 107 명(43.8\%) 순이었다. 학교 성적의 스트레스 요소는 학교과제 및 개설된 교육 과정이 182 명 $(74.6 \%)$ 로 가장 많았으며, 그 다음으 로는 개인적인 성격 28 명 $(11.5 \%)$, 경제 또는 사회 적 문제 9명 $(3.7 \%)$ 순으로 많았다.

<표 1>대상자의 일반적 특성 $(\mathrm{N}=244)$

\begin{tabular}{|c|c|c|c|}
\hline 특성 & 구분 & 빈도(븍 & 북분율) \\
\hline & 여성 & & $228(93.4)$ \\
\hline 성널 & 남성 & & $16(6.6)$ \\
\hline & $19-21$ & & $170(69.7)$ \\
\hline 연령 & $22-24$ & & $36(14.8)$ \\
\hline & $\geq 25$ & & $38(15.6)$ \\
\hline 연령 평균- & 표준편차 & & $22.24 \pm 3.09$ \\
\hline & 기독교 & & $125(51.2)$ \\
\hline & 불교 & & $97(39.8)$ \\
\hline 송피 & 천주교 & & $20(8.2)$ \\
\hline & 무 & & $2(0.8)$ \\
\hline & 미혼 & & $172(70.5)$ \\
\hline & 기혼 & & $72(29.5)$ \\
\hline & 매우 만족 & & $47(19.3)$ \\
\hline 자신의 & 만족 & & $156(63.9)$ \\
\hline 생활에 & 보통 & & $3(1.2)$ \\
\hline $\begin{array}{l}\text { 내안 } \\
\text { 마족 전도 }\end{array}$ & 불만 & & $33(13.5)$ \\
\hline & 매우 불만족 & & $5(2.0)$ \\
\hline & & 사전 & 사후 \\
\hline & 매우 우수(9점이상) & 7( 2.9) & 8(3.3) \\
\hline 학교성적 & 우수(6 8점) & $110(45.1)$ & 107(43.8) \\
\hline & 낮음(5 3점) & $122(50.0)$ & $124(51.3)$ \\
\hline & 매우 낮음(1 2점) & $5(2.0)$ & 5(1.6) \\
\hline 학교성적 & & & \\
\hline $\begin{array}{l}\text { 평균士 } \\
\text { 표준편차 }\end{array}$ & & $4.48 \pm .79$ & $4.99 \pm .78$ \\
\hline & 개인적인 성격 & & $28(11.5)$ \\
\hline & 건강상태 & & $7(2.9)$ \\
\hline & $\begin{array}{l}\text { 학교과제 및 } \\
\text { 개설된 교육과정 }\end{array}$ & & 182(74.6) \\
\hline & 종교적인 문제 & & $0(0.0)$ \\
\hline 스트레스 & 이성문제 & & $6(2.5)$ \\
\hline 요소 & 교우관계 & & $7(2.9)$ \\
\hline & 교수 & & $1(0.4)$ \\
\hline & 가족 & & $4(1.6)$ \\
\hline & $\begin{array}{l}\text { 경제 또는 } \\
\text { 사회적인 문제 }\end{array}$ & & $9(3.7)$ \\
\hline & 기타 & & $0(0.0)$ \\
\hline
\end{tabular}

2. 간호 시뮬레이션 교육이 간호대학생의 문 제해결과정과 자기효능감에 미치는 효과

간호 시뮬레이션 교육이 문제해결과정에 미치는 점수는 5점 만점에 실험처치 전에 3.38점, 실험처 치 후에 4.54점으로 간호대학생은 간호 시뮬레이션 교육을 전보다 실시한 후에 '문제해결과정'이 평균 평점이 1.16점이 높아졌으며, 통계적으로 유의한 차이를 보였다 $(\mathrm{t}=2.637, \mathrm{p}=.012)$. 문제해결과정의 5 가지 하부 단계를 분석한 결과 의사결정 $(\mathrm{t}=2.937$, $\mathrm{p}=.007)$, 해결책 수행 $(\mathrm{t}=2.589, \mathrm{p}=.016)$, 평가 및 반영 $(\mathrm{t}=2.453, \mathrm{p}=.023)$ 에서 통계적으로 유의한 차 이를 보였다.

간호 시뮬레이션 교육이 자기효능감에 미치는 점수는 5점 만점에 실험처치 전에 2.68점, 실험처 치 후에 2.72점으로 간호대학생은 간호 시뮬레이션 교육을 전보다 실시한 후에 '자기효능감'이 평균 0.04점이 높아졌으며, 통계적으로 유의한 차이를 보이지 않았다 $(\mathrm{t}=0.135, \mathrm{p}=.743)$. 자기효능감의 2 가 지 하부 단계를 분석한 결과 일반적 자기효능감 $(\mathrm{t}=0.591, \mathrm{p}=.543)$, 사회적 자기효능감 $(\mathrm{t}=0.047, \mathrm{p}=$ .964)으로 통계적으로 유의한 차이를 보이지 않았 다<표 2>.

3. 문제해결과정과 자기효능감간의 상관관계

간호 시뮬레이션 실습 교육 후에 간호대학생들 의 문제해결능력과 자기효능감간의 상관관계는 <표 3>와 같다. 문제해결능력과 자기효능감은 통 계적으로 유의한 정적인 상관관계를 보였다 $(\mathrm{r}=0.737, \mathrm{p}<.017)$. 결과적으로 문제해결과정이 높 을수록 자기효능감이 높은 것으로 나타났다. 
<표 2>간호 시뮬레이션 교육이 간호대학생의 문제해결과정과 자기효능감에 미치는 효과 $(\mathrm{N}=244)$

\begin{tabular}{|c|c|c|c|c|}
\hline & Pre test & Post test & \multirow{2}{*}{$t$} & \multirow{2}{*}{ r } \\
\hline & 평균土표준편차 & 평균土표준편차 & & \\
\hline 문제해결과정 & $3.38 \pm .33$ & $4.54 \pm .43$ & 2.637 & .012 \\
\hline 문제의 명료화 & $3.53 \pm 1.16$ & $4.03 \pm 1.12$ & 1.875 & .075 \\
\hline 해결방안모색 & $3.69 \pm .72$ & $4.08 \pm .68$ & 1.912 & .059 \\
\hline 의사결정 & $3.92 \pm 1.04$ & $4.54 \pm .82$ & 2.937 & .007 \\
\hline 해결책 수행 & $4.32 \pm 1.17$ & $4.91 \pm .84$ & 2.589 & .016 \\
\hline 평가 및 반영 & $4.48 \pm 1.07$ & $4.84 \pm .79$ & 2.453 & .023 \\
\hline 자기효능감 & $2.68 \pm .36$ & $2.72 \pm .30$ & 0.135 & .743 \\
\hline 일반적 자기효능감 & $2.65 \pm .72$ & $2.78 \pm .55$ & 0.591 & .543 \\
\hline 사회적 자기효능감 & $2.68 \pm .64$ & $2.73 \pm .42$ & 0.047 & .964 \\
\hline
\end{tabular}

<표 3> 문제해결능력, 자기효능감의 상관관계

\begin{tabular}{ccc}
\hline \multirow{2}{*}{ 구분 } & \multicolumn{2}{c}{ 자기효능감 } \\
\cline { 2 - 3 } & \multicolumn{2}{c}{$\mathrm{r}$} \\
\hline \hline 문제해결과정 & 0.737 & .017 \\
\hline
\end{tabular}

\section{$\mathrm{IV}$. 고찰 및 결론}

본 연구는 간호과 2 학년 대상으로 15 주간 간호 시뮬레이션 교육을 시행한 후 시뮬레이션 교육이 간호대학생의 문제해결과정과 자기효능감에 미치 는 영향을 파악하고자 시도되었다. 본 연구 결과에 서 간호시뮬레이션 교육전보다 교육 후에 학교성 적은 통계적으로 유의하지는 않았지만 미약하게 증가된 것은 시뮬레이션 교육에 의한 것이라기보 다 교과과정 중에 지식을 습득한 것으로 여겨진다. 한편 간호시뮬레이션 교육 사전시험은 이론중심에 서 평가되었다면 사후시험은 이론과 시뮬레이션 교육 후 평가하였으며 간호 시뮬레이션 실습이 팀 단위로 진행하였으므로 팀내에서 역할이 주어지지 않은 경우에는 경험하지 않을 수도 있으므로 사전 과 사후의 점수 차이가 적은 것으로 여겨진다.

간호 시뮬레이션 교육 시행 후 문제해결과정의 점수가 증가한 것으로 나타났다 $(\mathrm{t}=2.637, \mathrm{p}=.012)$.
문제해결과정은 5 개의 하위영역으로 분류되며 교 육 시행 전, 후의 차이가 가장 큰 항목은 의사결정 영역이며 문제의 명료화, 해결방안모색, 해결책 수 행, 평가 및 반영 영역은 교육 시행 전, 후 차이가 같은 것으로 나타났다. 간호시뮬레이션 교육은 실 제 간호 상황과 유사한 사례를 제시한 후 건강문 제를 모색하고 명료화하여 해결 방안을 모색하기 위해 의사결정을 촉진하게 된다. 본 연구에 참여한 학생들은 15 주간 이러한 학습 방법에 반복 노출되 어 학습함으로써 다양한 간호 문제를 호소하는 대 상자의 문제를 명료화하고 우선순위를 선정하고 우선순위별 해결방안을 모색하는 과정에서 토의하 고 결정하는 과정을 경험하고 학습함으로써 의사 결정 능력이 특히 촉진된 것으로 사료된다. 이우숙 등[11]의 성인의 문제해결과정 측정 도구 개발에서 학생들의 문제해결능력을 길러주기 위해서 일방적 주입방식인 전통적인 교사중심의 교수방법보다 학 생중심의 교수방법을 적용하는 것을 권장하고 있 으며 본 연구가 그 효과를 검증한 연구로 사료된 다. 이러한 결과는 문제중심학습이 문제해결과정에 유의한 향상을 가져온다는 유명란 등[23]의 연구와 시뮬레이션 연계 문제중심학습이 간호학생의 기본 역량에 미치는 효과에서 문제해결능력 향상을 가 져온다는 선행연구의 결과[24]와 유사하게 나타났 
다.

자기효능감은 개인이 어떤 결과를 산출하기 위 하여 필요한 행동을 성공적으로 수행할 수 있다고 느끼는 신념으로[17] 본 연구 결과에서 간호 시뮬 레이션 교육이 자기효능감에 미치는 효과를 살펴 보면, 참여 학생들의 일반적 자기효능감 정도는 교 육 전, 후 차이를 보였으나 통계적으로 유의한 차 이가 없는 것으로 나타났다. 이는 시뮬레이터를 활 용한 문제중심학습 모듈 개발 및 적용에 관한 단 일군 전후 설계 논문[25]에서 자기효능감이 통계적 으로 유의하지 않았다는 결과와 일치하였다. 한편 임상실습 교육에 관한 간호 학생의 자기효능감에 미치는 효과[16][25]에서는 자기효능감이 통계적으 로 유의한 것으로 나타났는데 이는 간호 시뮬레이 션 교육과 임상실습 교육 간의 학습방법의 차이로 사료된다. 자기효능감이 시뮬레이션실습과 임상실 습교육간의 차이가 있는 것은 간호시뮬레이션 교 육은 개별적인 과제를 제공하여 성공여부를 평가 하기보다는 팀단위로 이루어지는 교육과정이었으 므로 팀내에서 역할이 주어져서 수행할 경우에는 자기효능감이 높아질 수 있으나 팀내에서 역할이 주어지지 않았을 경우에는 자기효능감은 유의하지 않을 수 있으므로 이점을 고려하여 간호 시뮬레이 션 교육의 자기효능감은 신중하게 적용할 필요성 이 있는 것으로 사료된다. 그러므로 임상실습 교육 은 간호 술기에 대한 시범이나 설명을 주요 교육 방법으로 사용하므로 학생들이 듣고 살피는 인지 적 과정을 거쳐 모방에 의해 행동을 일으키게 되 므로 자기효능감 정도가 향상된 것으로 보인다. 이 에 반해 간호 시뮬레이션 교육은 문제중심학습으 로 간호 문제를 분석하고 우선순위별 해결방안을 모색하기 위해 의사결정하고 수행하고 평가하는 교육으로 자기주도성, 비판적 사고, 의사결정 능력 향상에 작용할 수 있지만 자기효능감에 영향이 적 게 주는 것으로 사료된다.

본 연구는 간호 시뮬레이션 교육이 간호대학생
의 문제해결과정과 자기효능감에 미치는 효과를 검증하기 위하여 시도된 단일군 전후 실험 설계이 고, 2010년 3월 2일부터 2010년 6월 11일까지 총 105 일간 U시에 있는 C대학 간호과 2학년 재학생 을 대상으로 하였다. 2010년 3월 2일에서 3월 9일 까지 실험처치 전 사전조사 하였으며. 실험처치로 시뮬레이션 실습을 2010년 3월 10일부터 2010년 6 월 2 일까지 주당 2 시간 15 주간 총 30 시간의 시뮬 레이션 실습을 운영하였다. 사후평가는 시뮬레이션 실습 종료 직후 2010년 6월 2일에서 6월 11일까지 진행하였다. 자료분석은 SPSS 12.0으로 분석하였으 며, 평균, 표준편차, paired t-test와 Cronbach's $\alpha$ 을 이용하여 분석하였다. 설문지는 대상자가 직접 설문을 읽고 응답하였으며, 설문지 작성에 소요된 시간은 10 20분간 소요되었다. 시뮬레이션 교육이 전·후 문제해결과정과 자기효능감에 미치는 효과 를 분석한 결과는 다음과 같이 나타났다.

1) 시뮬레이션 교육은 간호대학생의 문제해결과 정은 증가되었으므로 $(\mathrm{t}=2.637, \mathrm{p}=.012)$, 시뮬레이션 교육을 활용한 교육이 문제해결과정에 긍정적인 영향을 미치는 것을 알 수 있었다.

2) 시뮬레이션 교육은 자기효능감에는 통계적으 로 유의한 차이를 보이지 못하였으므로 $(\mathrm{t}=0.135$, $\mathrm{p}=.943)$, 시뮬레이션 교육이 간호대학생의 자기효 능감에 미치는 영향 미치지 못한 것으로 나타났다.

3) 간호 시뮬레이션 실습 교육 후에 간호대학생 들의 문제해결능력과 자기효능감간은 통계적으로 유의한 정적인 상관관계를 보였다 $(\mathrm{r}=0.737, \mathrm{p}$ <.017). 결과적으로 문제해결과정이 높을수록 자기 효능감이 높은 것으로 나타났다.

이상의 결과를 통하여 문제중심학습을 적용한 간호 시뮬레이션 교육은 문제해결과정에 긍정적인 영향을 미치지만 자기효능감에는 통계적으로 유의 한 차이가 없었다는 결과를 기초로 하여 향후 시 뮬레이션 실습교육의 효과를 극대화시키고 학습 성과를 이루는데 필요한 학습법을 개발하는데 기 
여할 것으로 사료된다. 현재 시뮬레이션 교육이 간 호 교육의 한 방안으로 적용되고 있는데 학습방법 에 따라 교육의 효과가 차이를 보이고 있어 향후 학습방법 간의 효과를 비교, 검증하는 추후 연구가 요구된다.

본 연구의 결과를 근거로 하여 다음과 같이 제 언하고자 한다.

첫째, 간호 시뮬레이션 교육은 문제해결능력을 증가시키므로 대조군과 실험군를 통해 문제해결 과정을 비교분석하여 시뮬레이션 교육의 구체적인 적용 방안을 모색할 필요가 있다.

둘째, 시뮬레이션 교육과정에 참여하였으나 실 습이 어려운 학생들을 대상으로 추가적인 교육 운 영이 요구된다.

셋째, 대조군과 실험군을 통해 학습방법에 따른 간호 시뮬레이션 교육의 효과를 비교, 검증하는 연 구가 요구된다.

\section{참고문헌}

1. 이애경, 김정애, 주미경, 정안순, 장은정, 김정수, 강정희, 이정애(2001), 임상실습 교육개선을 위한 간호학생의 간호활동시간 분석, 경북논총, Vol.5;523-535.

2. 양수, 이경주, 이소영(2006), 정신병동의 정신보건 간호사와 일반간호사의 문제해결 능력에 대한 자 기인식정도, 정신간호학회지, Vol.15(3);378-387.

3. 양승애, 정덕유(2004), 간호대학 학생의 비판적 사 고성향에 관한 조사연구, 성인간호학회지, Vol.16(1);156-165.

4. Nehring, W. N., Lashley, f. R.(2004), Current use and opinions regarding human patients simulators in nursing education: An international survey, Nursing Education Perspect, Vol.25(5);233-382.

5. Decker, S., Sportaman, S., Puetz, L., Billings, L.(2008), The evolution of simulation and its contribution to competency, Journal of Contin Education Nursing, Vol.39(2);74-80.

6. 유은영(2005), 의료시뮬레이션 의학강좌, 한국의사 회, pp.267-276.

7. 김소선(2008), 간호분야 실무 중심교육의 현황 및 전략, 국제학술심포지엄, 제주한라대학 PBL 교육 연구원, pp.33-39.

8. Lathrop, A., Winningham b., VandeVusse, L.(2007), Simulation-based learning for midwives: Background and pilot implementation, Journal of MidWifery Women Health, Vol.52(5);492-498.

9. Reilly, A., Spratt, C.(2007), The perceptions of undergraduate student nurses of high-fidelity simulation-based learning: A case report from the University of Tasmania, Nursing Education Today, Vol.27(6);542-550.

10. 조경애(2003), 협동학습과 개별학습이 문제해결과 정에 미치는 영향, 전북대학교 대학원 석사학위논 문, pp.9-22.

11. 이우숙, 박선환, 최은영(2008), 성인의 문제해결과정 측정도구 개발, 기본간호학회지, Vol.15(4);548-557.

12. 김영채(2002), 사고와 문제해결심리학, 박영사, pp.32-76.

13. 함영림(2010). 환자 시뮬레이터를 이용한 시뮬레 이션 교육 프로그램 개발 및 평가-간호학생을 대 상으로- 연세대학교 박사학위논문, pp.62-67.

14 김해란(2011). 간호학생을 위한 팀기반 시뮬레이 션 학습 프로그램 개발 및 효과, 조선대학교 박사 학위논문, pp.56-58.

15. 김해란, 최은영, 강희영, 김성민(2011), 시뮬레이 션기반 응급간호교육을 받은 간호학생의 학업성 취도와 자기효능감, 학습태도 및 수업만족도의 관 계, 한국간호교육학회지, Vol.17(1);5-13.

16. 김남희, 김경원(2011), 간호대학생의 임상실습교 육에 대한 자기효능감, 전문직 자아개념 및 임상 실습 만족도, 보건의료산업학회지 Vol.5(4);41-51.

17. 김정옥, 김현주, 조규영(2012), 수술실 간호사의 의료기술에 대한 인지와 자기효능감 및 전문직 
자아개념에 관한 연구, 보건의료산업학회지
Vol.6(1);1-13.

18. Bandura, A.(1977), Self-efficacy: Toward a unifying theory of behavioral change, Psychological Review, Vol.84(2);191-215.

19. Bandura, A.(1986), The Explanation and Predictive Scope of Self-Efficacy Theory, Journal of Social Clinic Psychology, Vol.4(3);359-373.

20. 한영인, 오혜경(2011), 핵심간호 시뮬레이션 교육, 수문사, pp.11-259.

21. Sherer M.S., Maddux J.E., Mercadante B., Prentice-Dunn S., Jacobs B., Rogers R.W.(1982), The self-efficacy scale: Construction and validation, Psychological report, Vol.51;663-671.

22. 홍혜영(1995), 완벽주의 성향, 자기효능감, 우울과 의 관계연구, 이화여자대학교 대학원 석사학위논 문, pp.18-64.

23. 유명란, 최윤정, 강명숙(2009), 문제중심학습이 학습 에 대한 자기주도성과 비판적 사고성향, 문제해결과 정에 미치는 효과, 기본간호학회지, Vol.16(1);46-55.

24. 이우숙, 조갑출, 양선희, 노영숙, 이규영(2009), 시뮬 레이션 연계 문제중심학습이 간호학생의 간호기본역 량에 미치는 효과, 기본간호학회지, Vol.16(1);64-72.

25. 주민선, 황윤영, 박창승(2006), 시뮬레이터를 활용 한 문제중심학습 모듈 개발 및 적용 -simman을 중심으로-, 기본간호학회지 Vol.13(2);182-189. 